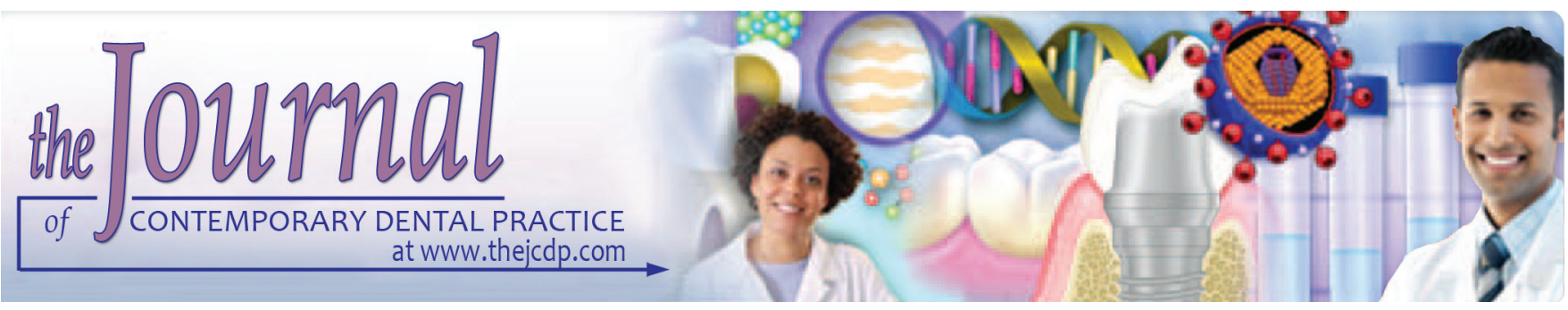

\title{
Oral Manifestations in Diabetic and Nondiabetic Chronic Renal Failure Patients receiving Hemodialysis
}

\author{
${ }^{1}$ Ravi Dande, ${ }^{2}$ Amol R Gadbail, ${ }^{3}$ Sheetal Sarode, ${ }^{4}$ Mugdha P Mankar Gadbail, ${ }^{5}$ Shailesh M Gondivkar \\ ${ }^{6}$ Madhuri Gawande, ${ }^{7}$ Sachin C Sarode, ${ }^{8}$ Gargi S Sarode, ${ }^{9}$ Shankargouda Patil
}

\section{ABSTRACT}

Aim: To assess the prevalence of oral findings in diabetic and nondiabetic chronic renal failure $(\mathrm{CRF})$ patients receiving hemodialysis (HD).

Materials and methods: This study was conducted on $144 \mathrm{CRF}$ patients undergoing HD therapy. They were further classified into diabetic and nondiabetic groups and also according to the duration of CRF. An oral examination was performed, searching for CRF-associated oral findings, along with due considerations to duration of CRF.

Results: The oral manifestations were found to be higher in diabetic CRF patients $(97.14 \%)$ relative to nondiabetic CRF patients (89.18\%). The diabetic group exhibited a significantly higher number of patients with poor oral hygiene index $(p=000)$, uremic fetor $(p=0.005)$, unpleasant taste $(p=0.009)$, dry-fissured lips $(p=0.002)$, and pale mucosa $(p=0.019)$ than the nondiabetic

\footnotetext{
1,2Department of Dentistry, Indira Gandhi Government Medical College \& Hospital, Nagpur, Maharashtra, India

${ }^{3}$ Sharad Pawar Dental College \& Hospital, Datta Meghe Institute of Medical Sciences, Wardha, Maharashtra, India

${ }^{4}$ Department of Orthodontia, Government Dental College \& Hospital, Nagpur, Maharashtra, India

${ }^{5}$ Department of Medicine and Radiology, Government Dental College \& Hospital, Nagpur, Maharashtra, India

${ }^{6}$ Department of Oral and Maxillofacial Pathology \& Microbiology Sharad Pawar Dental College \& Hospital, Datta Meghe Institute of Medical Sciences, Wardha, Maharashtra, India

${ }^{7,8}$ Department of Oral \& Maxillofacial Pathology, Dr. D. Y. Patil Dental College \& Hospital, Dr. D. Y. Patil Vidyapeeth, Pune Maharashtra, India

${ }^{9}$ Division of Oral Pathology, Department of Maxillofacial Surgery and Diagnostic Sciences, College of Dentistry, Jazan University Jazan, Kingdom of Saudi Arabia

Corresponding Author: Amol R Gadbail, Department of Dentistry, Indira Gandhi Government Medical College \& Hospital, Nagpur, Maharashtra, India, e-mail: gadbail@yhoo. co.in/argadbail@gmail.com
}

group. The oral manifestations were significantly higher in CRF patients of more than 24 months' duration.

Conclusion: The CRF HD patients with diabetes mellitus exhibited a higher risk of oral uremic manifestations including uremic fetor, unpleasant taste, pale mucosa, and dry-fissured lips than nondiabetics. The oral health status of CRF HD patients becomes worse with the increase in the duration of CRF.

Clinical significance: Oral health status is often a neglected aspect in CRF patients. It should be given prime importance for CRF patients receiving HD to improve the quality of life.

Keywords: Chronic renal failure, Diabetes, Hemodialysis, Oral manifestations.

How to cite this article: Dande R, Gadbail AR, Sarode S, Gadbail MPM, Gondivkar SM, Gawande M, Sarode SC, Sarode GS, Patil S. Oral Manifestations in Diabetic and Nondiabetic Chronic Renal Failure Patients receiving Hemodialysis. J Contemp Dent Pract 2018;19(4):398-403.

Source of support: (STS Funding) ICMR, New Delhi, India

Conflict of interest: None

\section{INTRODUCTION}

Chronic renal failure is the result of gradual reduction of functional nephrons which results in functions loss of kidney. There are various possible causes, ${ }^{1}$ and the natural course of CRF leads to terminal or end-stage renal disease, which is the final syndrome for several primary renal diseases, and systemic diseases, causing kidney function loss. ${ }^{2,3}$ The CRF is predominantly an adult disease, while affecting all age groups. Diabetes mellitus is an important risk factor for CRF. Diabetic patients are at risk for acute and chronic complications in oral cavity, such as xerostomia, glossodynia, bacterial, viral, and fungal infections, especially oral candidiasis, and periodontal disease. ${ }^{4-6}$

Up to $90 \%$ prevalence of oral manifestations has been reported in end-stage renal disease patients. At least one 
or more than 30 different oral signs and symptoms were associated with CRF and kidney transplant including xerostomia, uremic fetor, pale mucosa, uremic stomatitis, and candidiasis. ${ }^{7}$ The aim of this study was to assess the prevalence of oral signs and symptoms in a group of diabetic and nondiabetic CRF patients, along with due considerations to duration of CRF.

\section{MATERIALS AND METHODS}

The study was carried out at Acharya Vinoba Bhave Rural Hospital at Sawangi (M), Wardha, Maharashtra, India. This study was conducted on CRF patients undergoing HD therapy in the inpatient department and day-care. The ethical approval was obtained from the institutional ethical committee of Datta Meghe Institute of Medical Sciences, Deemed University, Wardha, Maharashtra, India. Totally 144 patients were examined and an informed consent was obtained. Patients having any other systemic illness, other than hypertension and diabetes mellitus, were excluded from this study. Data including demographic data, type of dialysis, duration of CRF, hypertension, and diabetes mellitus were collected. A detailed drug history was asked for hypertensive patients and diabetic patients. Then selected patients of CRF were subjected to biochemical investigations, mainly serum creatinine and creatinine clearance. Patients with a serum creatinine $\geq 2.0 \mathrm{mg} / \mathrm{dL}$, creatinine clearance $\leq 60 \mathrm{~mL} / \mathrm{min}$, and glomerular filtration rate $\leq 50 \%$ were included in this study. Intraoral examination was done for oral mucosal lesions. Signs and symptoms for the CRF patients were objectively asked and/or reported by patients. For the patients who gave a history of dry mouth along with a dry or sticky mucosa, a diagnosis of xerostomia was made. The patients were subsequently subjected to modified Schirmer's test to measure the unstimulated salivary flow. ${ }^{8}$ Uremic fetor was identified when the patient had a urine-odor breath, whereas an unpleasant taste was considered when the patient had a history of altered taste sensation. Oral hygiene status was evaluated by using the simplified oral hygiene index by Greene and Vernillion, ${ }^{9}$ and gingival status (i.e., gingivitis) was evaluated by using the papilla bleeding index by Muhlemann. ${ }^{10}$ The periodontal status was recorded by recording the clinical attachment level (CAL) (i.e., periodontitis if CAL $>1 \mathrm{~mm}$ ), gingival recession, probing pocket depth, mobility, and furcation involvement. Acknowledged clinical diagnosis criteria for CRF-associated oral manifestations ${ }^{7,11}$ were used to diagnose the oral lesions, and all the findings were recorded. To classify the degree of medical control, the CRF patients were subdivided into three groups according to the duration as less than 12 months (group I), 12 to 24 months (group II), and more than 24 months (group III).

\section{Statistical Analysis}

Descriptive statistics including frequencies, percentages, and means along with standard deviation (SD) were calculated. Inter- and intragroup variable associations were analyzed with Pearson chi-square and Fisher's exact tests. Age, CRF, diabetes, and dialysis duration were compared with Student's t test. Statistical analyses were performed using the statistical software package Statistical Package for the Social Sciences version 16.0 (SPSS, Chicago, Illinois); $\mathrm{p}<0.05$ was considered statistically significant.

\section{RESULTS}

One hundred forty-four CRF patients including 108 males $(75.00 \%)$ and 36 females $(25.00 \%)$ undergoing maintenance HD therapy were included in the present study. The demographics of the diabetic and nondiabetic CRF patients are provided in Table 1 . There were no statistically significant differences between the diabetic and nondiabetic groups with regard to age, gender, smokers, and CRF duration at baseline (Table 1).

The distributions of oral manifestations for both the groups are shown in Table 2. The group of diabetic CRF patients (97.14\%) exhibited a higher prevalence of oral signs and symptoms relative to nondiabetic group $(89.18 \%)$. Poor oral hygiene $(70.83 \%)$, uremic fetor

Table 1: Demographic distributions and general health behaviors between diabetic and nondiabetic CRF patients receiving HD at baseline

\begin{tabular}{llllll}
\hline \multirow{2}{*}{ Characteristics } & & Total $(n=144)$ & $\begin{array}{l}\text { Nondiabetic CRF } \\
\text { patients }(n=74)\end{array}$ & $\begin{array}{l}\text { Diabetic CRF } \\
\text { patients }(n=70)\end{array}$ & $p$-value \\
\hline Age & Mean & 47.87 & 46.67 & 49.14 & 0.150 \\
& SD & 11.83 & 11.76 & 11.94 & 0.077 \\
Gender & Male & $108(75.00 \%)$ & $62(83.78 \%)$ & $46(65.71 \%)$ & $24(34.28 \%)$ \\
& Female & $36(25.00 \%)$ & $12(16.21 \%)$ & $20(28.57 \%)$ & 0.002 \\
Hypertension & & $68(47.22 \%)$ & $48(64.86 \%)$ & 28.60 & 0.891 \\
Duration of CRF with HD & Mean & 26.93 & 25.35 & 14.43 & 0.277 \\
& SD & 14.35 & 14.30 & $6(9.37 \%)$ & 0.017 \\
Smoker & & $8(5.55 \%)$ & $2(2.70 \%)$ & $44(62.85 \%)$ & \\
Tobacco chewer & & $66(45.83 \%)$ & $22(29.72 \%)$ & & \\
\hline
\end{tabular}


Table 2: Oral manifestations in diabetic and nondiabetic CRF patients receiving HD

\begin{tabular}{lllll}
\hline Oral manifestations & Total $(n=144)$ & $\begin{array}{l}\text { Diabetic CRF } \\
\text { patients }(n=70)\end{array}$ & $\begin{array}{l}\text { Nondiabetic CRF } \\
\text { patients }(n=74)\end{array}$ & $\begin{array}{l}\text {-value } \\
\text { Poor OHI }\end{array}$ \\
Ulcers in mouth & $102(70.83 \%)$ & $62(88.57 \%)$ & $6(8.10 \%)$ & 0.000 \\
Dryness in mouth & $12(8.33 \%)$ & $6(8.77 \%)$ & $36(48.64 \%)$ & 0.943 \\
Gingivitis & $78(54.66 \%)$ & $42(60.00 \%)$ & $18(25.71 \%)$ & 0.334 \\
Periodontitis & $42(29.16 \%)$ & $24(32.43 \%)$ & $10(13.51 \%)$ & 0.531 \\
Uremic fetor & $28(19.44 \%)$ & $18(25.71 \%)$ & $44(59.45 \%)$ & 0.191 \\
Dry-fissured lips & $103(73.61 \%)$ & $62(88.57 \%)$ & $2(2.70 \%)$ & 0.005 \\
Pale mucosa & $22(15.27 \%)$ & $20(28.57 \%)$ & $26(35.13 \%)$ & 0.002 \\
Unpleasant taste & $70(48.61 \%)$ & $44(62.85 \%)$ & $26(35.13 \%)$ & 0.019 \\
\hline
\end{tabular}

OHI: Oral hygiene index

Table 3: Correlations between duration of CRF and oral manifestations

\begin{tabular}{llllll}
\hline & & \multicolumn{3}{c}{ Duration of CRF } \\
\cline { 3 - 5 } Oral manifestations & Total $(n=144)$ & Group I $(n=36)$ & Group II $(n=46)$ & Group III $(n=62)$ & $p$-value \\
\hline Poor OHI & 102 & $17(47.22 \%)$ & $32(69.56 \%)$ & $53(85.48 \%)$ & 0.000 \\
Ulcers in mouth & 12 & $4(11.11 \%)$ & $6(13.04 \%)$ & $2(03.22 \%)$ & 0.385 \\
Dryness of mouth & 78 & $14(38.88 \%)$ & $28(60.86 \%)$ & $36(58.06 \%)$ & 0.317 \\
Gingivitis & 42 & $16(44.44 \%)$ & $8(17.39 \%)$ & $18(29.04 \%)$ & 0.167 \\
Periodontitis & 28 & $12(33.33 \%)$ & $6(13.04 \%)$ & $10(16.12 \%)$ & 0.219 \\
Uremic fetor & 106 & $12(16.66 \%)$ & $34(73.19 \%)$ & $60(96.77 \%)$ & 0.000 \\
Dry-fissured lips & 22 & $0(00.00 \%)$ & $4(8.69 \%)$ & $18(29.03 \%)$ & 0.014 \\
Pale mucosa & 70 & $4(11.11 \%)$ & $16(34.78 \%)$ & $50(80.64 \%)$ & 0.000 \\
Unpleasant taste & 72 & $2(5.55 \%)$ & $16(34.78 \%)$ & $54(87.09 \%)$ & 0.000 \\
\hline
\end{tabular}

OHI: Oral hygiene index (3.1-6.0); group I = duration of CRF less than 12 months; group II = duration of CRF less than 12 to 24 months; group III = duration of CRF more than 12 months

(73.61\%) followed by dryness of the mouth $(54.66 \%)$, unpleasant taste $(50.00 \%)$, and pale mucosa $(48.61 \%)$ were the most frequently noticed findings in these CRF patients. Other less commonly noted manifestations were gingivitis $(29.16 \%)$, periodontitis $(19.44 \%)$, dry-fissured lips (15.27\%), and oral ulcerations (8.33\%). Among these reported oral mucosal findings, the diabetic group revealed significantly higher levels of poor oral hygiene $(p=0.000)$, uremic fetor $(p=0.005)$, unpleasant taste $(p=0.009)$, dry-fissured lips $(p=0.002)$, and pale mucosa $(p=0.019)$ than the nondiabetic group. Oral ulcerations and dryness of the mouth (xerostomia) were not significantly different between the two groups. Gingivitis $(p=0.531)$ and periodontitis $(p=0.191)$ were also significantly not different in both groups; however, they tended to be slightly higher in the diabetic group than in the nondiabetic group.

The oral manifestations noticed according to duration of CRF are shown in Table 3. On comparing groups I, II, and III, oral manifestations that were observed with highest prevalence among the patients with CRF for more than 24 months duration (group III) and with lowest prevalence among those with CRF with less than 12 months of duration (group I) were poor oral hygiene $(p=0.000)$, uremic fetor $(p=0.000)$, unpleasant taste $(p=0.000)$, pale mucosa $(p=0.000)$, and dry-fissured lips $(p=0.014)$. The duration of CRF was not found to be significantly associated with other manifestations including oral ulceration $(p=0.385)$, dryness of mouth $(p=0.317)$, gingivitis $(p=0.167)$, and periodontitis $(p=0.219)$.

\section{DISCUSSION}

The CRF is usually irreversible and progressive in nature and occurs when the glomerular filtration rate is around 5 to $10 \%$ associated with a high level of uremia. ${ }^{12}$ The physiological and biochemical abnormality of serious renal failure leads to specific signals and symptoms. Multiple complications are noted in CRF patients due to the influence of etiology, decreased renal function, response to treatment, and other associated systemic illness. ${ }^{13}$

The complete biochemical investigation was not carried out; however, the present data obtained by the clinical investigation were compared well. The present study examined and compared the oral signs and symptoms in diabetic and nondiabetic CRF patients receiving $\mathrm{HD}$, along with due considerations to duration of CRF. The present study results revealed that diabetic CRF patients on HD had more prevalence of oral manifestations and poor oral hygiene than nondiabetic CRF patients. Almost $97.14 \%$ of diabetic CRF patients had 
either one or more symptoms or signs, the most prevalent being poor oral hygiene, uremic fetor, unpleasant taste, pale mucosa, and dry-fissured lips. In this study, $70.83 \%$ of participants had poor oral hygiene. In previous studies, it was reported that the oral hygiene status of patients having chronic kidney disease is poor ${ }^{14,15}$ due to the negligence of their oral health as the disease progresses due to stress, xerostomia, gingival hyperplasia, malnutrition, and drugs, such as antidepressants and anticoagulants. The results of this study indicate that as the duration of CRF progresses, the status of poor oral hygiene increases. ${ }^{14}$ A poor oral hygiene was seen more in the diabetic group of CRF patients as compared with the nondiabetic group of CRF patients.

Xerostomia was clinically manifested by the dryness of mouth and sensation of thirst. In the present study, the prevalence of the dryness of mouth was observed in $54.66 \%$ CRF patients, which is in agreement with other studies of dialysis patients. ${ }^{16,17}$ The aggravation of discomfort and anxiety was observed in HD patients, which may be due to their limited water intake. ${ }^{18}$ In addition, severe thirst resulted in interdialysis body weight gain caused due to undue water intake, which may increase the risk of elevated blood pressure and heart failure. ${ }^{19}$ Other reasons may be the side effects of drugs, possible salivary gland alteration, and oral breathing secondary to lung perfusion problems. ${ }^{7,18}$ The prevalence of dryness of mouth in diabetic $(60.00 \%)$ CRF patient was higher than in nondiabetics (48.64\%). Minor salivary glands atrophy or dysfunction that occurs due to involvement by micro vascular disease and neuropathy explains the high prevalence of xerostomia in diabetic CRF patients ${ }^{4,5}$ Xerostomia in the diabetic CRF patient may lead to further complications by acting as a risk factor for candidiasis, dental caries, periodontal disease, and bacterial infections. ${ }^{17,20}$

The prevalence of uremic fetor in the diabetic group $(88.57 \%)$ was higher than in the nondiabetic group (59.45\%). A high urea concentration in saliva, and its consequent conversion to ammonia, leads to uremic fetor. ${ }^{21}$ The measurement of salivary urea was not included in this study to support this assumption; however, it will be further investigated. The $73.61 \%$ uremic fetor found in CRF patients in this study is in agreement with the study by Kao-Ch et al. ${ }^{22}$ In the present study, the unpleasant taste was observed in $50.00 \%$ of CRF patients. An altered perception to sweet and sour taste was noted in patients with CRF. ${ }^{23}$ Impairment in taste modalities is usually seen in younger patients than older patients, but they may have a better recovery of neural taste function following dialysis. ${ }^{24}$ The prevalence of unpleasant taste was observed higher in the diabetic group (65.71\%) than in the nondiabetic CRF patients (35.13\%). Additional possible causes are increased phosphate and protein concentrations and changes in salivary $\mathrm{pH}$, which might explain a metallic or unpleasant taste. ${ }^{25}$

In this study, oral ulceration was noted only in $8.33 \%$ of CRF patients with a nonsignificant difference between the diabetic and nondiabetic groups. Ulcerations in the mouth encountered are probably because of decreased salivary secretions as well as splitting of urea by the commensals present in the oral cavity. ${ }^{26}$ Paleness of the mucosal membranes due to anemia, ${ }^{27}$ a more common complication in the CRF patient, may be caused by folic acid and erythropoietin deficiencies, inhibited erythropoiesis along with shortened erythrocyte life span, increase hemolysis as result of HD complications. ${ }^{8,23}$

Recent studies focusing on the periodontal health of CRF patients on HD maintenance therapy have reported that the presence of poor oral hygiene, gingival, and periodontal inflammation. ${ }^{28,29}$ Similarly, in the present study, poor oral hygiene, gingivitis, and periodontitis were observed in 70.83, 29.16, and $19.44 \%$ cases of CRF respectively. The increased incidence of gingival inflammation universally reported in renal HD populations might be due to several possible reasons. Most prominently, CRF patients on HD develop the uremic syndrome resulting into uremia, which has been associated with immune dysfunction, mainly defects in lymphocyte and monocyte function. ${ }^{28}$ Therefore, if uremia is responsible for the poor periodontal status observed in this population, increased dialysis vintage maintenance therapy should be carried out in CRF patients with increased incidence and severity of gingivitis and periodontitis. Increased gingival inflammation and periodontitis have been reported in association with increased dialysis vintage in several, ${ }^{27-30}$ but not all studies. ${ }^{31-33}$

In addition to uremia, the presence of confounding diseases, such as diabetes mellitus could contribute to the increased gingival inflammation reported for renal HD populations. Especially in view of diabetes mellitus being the most common cause for CRF populations a strong association was noted between diabetes mellitus and periodontitis. ${ }^{34}$ In the present study, gingivitis and periodontitis are not significantly different in diabetic and nondiabetic CRF patients on HD, which is in agreement with the results of previous study. ${ }^{35}$

The limitations of this study were that no physiological or biochemical systemic changes were measured. However, the increased duration of CRF patients with HD may worsen the physiological and biochemical systemic abnormalities. We observed a significantly increased prevalence of uremic fetor, dry-fissured lips, pale mucosa, and unpleasant taste with an increase in the duration of CRF on HD. Dryness in the mouth was not significantly increased with the duration of CRF on HD. However, 35.89 and $46.15 \%$ out of 39 patients with dryness of mouth 
were observed in CRF patient with a duration of 12 to 24 months and more than 24 months respectively.

\section{CONCLUSION}

The results suggest that CRF HD patients with diabetes mellitus exhibited a higher risk of oral uremic manifestations including uremic fetor, unpleasant taste, pale mucosa, and dry-fissured lips than nondiabetics. The oral health status of CRF HD patients becomes worse with the increase in the duration of CRF. Thus, oral health maintenance is of utmost importance in this patient group. The oral health maintenance awareness program should be reinforced for CRF patients on HD by the dialysis team and the dentists. Otherwise, oral pathologies and infections could be attributed as risk factors for acute bacterial endocarditis and jeopardize the opportunity to receive a successful kidney transplant. Further, a study should be carried out to clarify the effect of dialysis in diabetic patients by using a larger cohort of patients and with full clinical oral examination, such as dental status, saliva samples, and X-rays.

\section{REFERENCES}

1. Snyder S, Pendergraph B. Detection and evaluation of chronic kidney disease. Am Fam Physician 2005 Nov;72(9):1723-1732.

2. Sobrado-Marinho JS, Tomas Carmona I, Loureiro A, Limeres Posse J, Garcia Caballero L, Diz Dios P. Oral health status in patients with moderate-severe and terminal renal failure. Med Oral Patol Oral Cir Bucal 2007 Aug;12(4):305-310.

3. Gudapati A, Ahmed P, Rada R. Dental management of patients with renal failure. Gen Dent 2002 Nov-Dec;50(6):508-510.

4. Manfredi M, McCullough MJ, Vescovi P, Al-Kaarawi ZM, Porter SR. Update on diabetes mellitus and related oral diseases. Oral Dis 2004 Jul;10(4):187-200.

5. Arrieta-Blanco JJ, Bartolome-Villar B, Jimenez-Martinez E, Saavedra-Vallejo P, Arrieta-Blanco FJ. Bucco-dental problems in patients with diabetes mellitus (1): index of plaque and dental caries. Med Oral 2003 Mar-Apr;8(2):97-109.

6. Molina SD, Monroy RE, Arenas R, Fernandez RF, Rubalcaba PJ, Fabian SMG. Frecuencia de candidiasis oral en pacientes diabeticos tipo 2 ambulatorios en el Hospital General Manuel Gea Gonzalez. Estudio clínico micológico. Dermatología Rev Mex 2002;46:3-9.

7. Proctor R, Kumar N, Stein A, Moles D, Porter S. Oral and dental aspects of chronic renal failure. J Dent Res 2005 Mar;84(3):199-208.

8. Kumar NN, Panchaksharappa MG, Annigeeri RG. Modified Schirmer test-a screening tool for xerostomia among subjects on antidepressants. Arch Oral Biol 2014 Aug;59(8):829-834.

9. Greene JC, Vermillion JR. The simplified oral hygiene index. J Am Dent Assoc 1964 Jan;68:7-13.

10. Muhlemann HR. Psychological and chemical mediators of gingival health. J Prev Dent 1977 Jul-Aug;4(4):6-16.

11. Cohen SG. Renal disease. In: MA Lynch, VJ Brightman, MS Greenberg, editors Burket's Oral Medicine. Mexico: McGrawHill International; 1996. pp. 492-514.
12. Bottomley WK, Cioffi RF, Martin AJ. Dental management of the patient treated by renal transplantation: preoperative and postoperative considerations. J Am Dent Assoc 1972 Dec;85(6):1330-1335.

13. Kalyvas D, Tosios KL, Leventis MD, Tsiklakis K, Angelopoulos AP. Localized jaw enlargement in renal osteodystrophy: report of a case and review of the literature. Oral Surg Oral Med Oral Pathol Oral Radiol Endod 2004 Jan;97(1):68-74.

14. Tadakamadla J, Kumar S, Mamatha GP. Comparative evaluation of oral health status of chronic kidney disease (CKD) patients in various stages and healthy controls. Spec Care Dentist 2014 May-Jun;34(3):122-126.

15. de Souza CM, Braosi AP, Luczyszyn SM, Olandoski M, Kotanko P, Craig RG, Trevilatto PC, Pecoits-Filho R. Association among oral health parameters, periodontitis, and its treatment and mortality in patients undergoing hemodialysis. J Periodontol 2014 Jun;85(5):e169-e178.

16. Kho HS, Lee SW, Chung SCh, Kim YK. Oral manifestations and salivary flow rate, $\mathrm{pH}$, and buffer capacity in patients with end-stage renal disease undergoing hemodialysis. Oral Surg Oral Med Oral Pathol Oral Radiol Endod 1999 Sep;88(3):316-319.

17. Postorino M, Catalano C, Martorano C, Cutrupi S, Marino C, Cozzupoli P, Scudo P, Zoccali C. Salivary and lacrimal secretion is reduced in patients with ESRD. Am J Kidney Dis 2003 Oct;42(4):722-728.

18. Oldenburg B, Macdonald GJ, Perkins RJ. Factors influencing excessive thirst and fluid intake in dialysis patients. Dial Transplant 1988;17:21-23.

19. Charra B, Chazot C. Volume control, blood pressure and cardiovascular function. Lessons from hemodialysis treatment. Nephron Physiol 2003;93(4):94-101.

20. Kaya M. Cermik TF, Üstun F, Sen S, Berkarda S. Salivary function in patients with chronic renal failure undergoing hemodialysis. Ann Nuclear Med 2002 Apr;16(2):117-120.

21. Epstein SR, Mandel I, Scopp IW. Salivary composition and calculus formation in patients undergoing hemodialysis. J Periodontol 1980 Jun;51(6):336-338.

22. Kao ChH, Hsieh JF, Tsai SCh, Ho YJ, Chang HR. Decreased salivary function in patients with end-stage renal disease requiring hemodialysis. Am J Kidney Dis 2000 Dec;36(6): 1110-1114.

23. Shepherd R, Farleigh CA, Atkinson C, Pryor JS. Effects of haemodialysis on taste and thirst. Appetite 1987 Oct;9(2): 79-88.

24. Ciechanover M, Peresecenschi G, Aviram A, Steiner JE. Malrecognition of taste in uremia. Nephron 1980;26(1): 20-22.

25. Skorecki K, Green J, Brenner BM. Chronic renal failure. In: DL Kasper, E Braunwald, AS Fauci, SL Hauser, DL Longo, JL Jameson, editors Harrison's principles of internal medicine. New York: McGraw-Hill; 2005. pp. 1653-1663.

26. Baney GPE. Oto-laryngeal problems arising during the management of severe renal failure. J Laryngol Otol 1964 May;78:507.

27. Davidovich E, Davidovits M, Eidelman E, Schwarz Z, Bimstein E. Pathophysiology, therapy, and oral implications of renal failure in children and adolescents: an update. Pediatr Dent 2005 May-Apr;27(2):98-106.

28. Duran I, Erdemir EO. Periodontal treatment needs of patients with renal disease receiving haemodialysis. Int Dent J 2004 Oct;54(5):274-278. 
29. Chen L-P, Chiang C-K, Chan C-P, Hung K-Y, Huang C-S. Does periodontitis reflect inflammation and malnutrition status in hemodialysis patients? Am J Kidney Dis 2006 May;47(5):815-822.

30. Cohen G, Haag-Weber M, Horl WH. Immune dysfunction in uremia. Kidney Int 1997 Nov;62:S79-S82.

31. Naugle K, Darby ML, Bauman DB, Lineberger LT, Powers R. The oral health status of individuals on renal dialysis. Ann Periodontol 1998 Jul;3(1):197-205.

32. Al-Wahadni A, Al-Omari MA. Dental diseases in a Jordanian population on renal dialysis. Quintessence Int 2003 May;34(5): 343-347.
33. Marakoglu IK, Gursoy KD, Demirer S, Sezer H. Periodontal status of chronic renal failure patients receiving hemodialysis. Yonsei Med J 2003 Aug;44(4):648-652.

34. GrossiSG,ZambonJJ,HoAW,Koch G, Dunford RG, Machtei EE, Norderyd OM, Genco RJ. Assessment of risk for periodontal disease. Risk indicators for attachment loss. J Periodontol 1994 Mar;65(3):260-267.

35. Chuang SF, Sung JM, Kuo SC, Huang JJ, Lee SY. Oral and dental manifestations in diabetic and non-diabetic uremic patients receiving hemodialysis. Oral Surg Oral Med Oral Pathol Oral Radiol Endod 2005 Jun;99(6):689-695. 